\title{
La toma de conciencia de los estudiantes de educación musical en los cursos de lenguaje y percepción musical
}

\author{
The awareness of musical education students in the courses of language \\ and musical perception
}

\author{
Julio Salas González \\ jasggaiter@gmail.com \\ Código ORCID: 0000-0002-0660-2120 \\ Universidad Pedagógica Experimental Libertador, Venezuela
}

\section{RESUMEN}

- Artículo recibido en octubre 2019

El propósito de la presente investigación fue analizar las vivencias de los estudiantes de la especialidad de Educación Musical en los cursos de Lenguaje y Percepción Musical, para luego reflexionar sobre la toma de conciencia de ellos, producto de sus vivencias en relación con las vías pedagógicas necesarias para el desarrollo de la audiopercepción. La metodología se enmarcó dentro del enfoque cualitativo, sustentado en el método fenomenológico trascendental. Como resultados se obtuvieron: a) los estudiantes deben centrar sus formas de estudio en procesos reflexivos que busquen la consolidación de experiencias significativas; b) la importancia de un proceso de pedagogía musical en contraposición a la enseñanza tradicional, que busque la creación de espacios pedagógicos lúdicos. En fin, la clave está en el desarrollo de la audiopercepción, basada en tres conceptos esenciales: pedagogía de la música, práctica desde la experiencia y el docente mediador.

Palabras clave: Lenguaje musical; percepción música; toma de conciencia

\begin{abstract}
The purpose of the present investigation was to analyze the experiences of the students of the specialty of Musical Education in the courses of Language and Musical Perception, to later reflect on their awareness, product of their experiences in relation to the necessary pedagogical pathways for the development of audio perception. The methodology was framed within the qualitative approach, based on the transcendental phenomenological method. As results were obtained: a) students should focus their forms of study on reflective processes that seek to consolidate significant experiences; $b$ ) the importance of a process of musical pedagogy as opposed to traditional teaching, which seeks the creation of recreational educational spaces. In short, the key is in the development of audioperception, based on three essential concepts: music pedagogy, practice from experience and the mediating teacher.
\end{abstract}

Key words: Musical language, musical perception, awareness 


\section{INTRODUCCIÓN}

En los procesos de enseñanza y aprendizaje en el área musical aún sigue siendo un problema actual el aprendizaje del solfeo y el desarrollo de la audiopercepción, ya que son notorias amplias debilidades de los estudiantes al momento de la práctica individual de ejercicios de lectura y solfeo entonado, a la gran mayoría de los estudiantes se les dificulta el logro de una afinación precisa así como el reconocimiento y la discriminación de cada nota musical que forme parte de una estructura rítmico-melódica; lo que se traduce en motivo de frustración.

Esta problemática es en un gran porcentaje debido a que la mayoría de los estudiantes inician su formación educativa musical sin poseer experiencias previas, pues aunque no es requisito primordial, es bien sabido que quienes recibieron una formación musical previa y no formal antes de su escolarización muestran avances significativos con más rapidez. Sin embargo es necesario resaltar que otro factor influyente en las debilidades durante el desarrollo de la audiopercepción y la entonación es un modelo de formación basado en una constante memorización de lecciones mediante la repetición, en donde la recirculación de la información es únicamente la estrategia de aprendizaje potenciada. De esta manera predomina en la enseñanza de la música un enfoque positivista, donde el facilitador deja de lado escenarios de aprendizaje que potencien el pensamiento reflexivo y los procesos metacognitivos

De esta manera se palpa la necesidad de un profesor de música capaz de crear verdaderos espacios constructivos, estructurados en modelos pedagógicos estratégicos, que permitan la formación de un estudiante crítico, capaz de entender su contexto y el mundo que lo rodea desde una perspectiva sonora perceptible. De esta manera, las vivencias de cada uno de los estudiantes, serán la razón de ser de la construcción de su aprendizaje, según las necesidades de cada uno de ellos.

\section{MÉTODO}

Debido a que la presente investigación pretendía comprender las evidencias surgidas a través de la conciencia despierta y las vivencias de los informantes, se seleccionó como método de investigación a la fenomenología trascendental, la cual según Husserl (1949) se mueve en todos sus pasos dentro de los ámbitos de la conciencia refleja. Mediante ello todas las formas de la experiencia que se desarrollan en el fluir de la vida se convierten en el objeto de una nueva consideración. Además Husserl (1949) también mantiene que todo acto de reflexión, cualquiera que sea su grado, tiene en último término en su base un acto directo que se dirige naturalmente a la "trascendencia". 
Los informantes estuvieron representados por estudiantes de la especialidad Educación Musical del Instituto Pedagógico de Miranda de la Universidad Pedagógica Experimental Libertador (UPEL), específicamente, aquellos que han cursado y aprobado los cursos de Lenguaje y Percepción Musical I y Lenguaje y Percepción Musical II.

La recolección de la información dentro de las investigaciones cuyo método lo constituye la fenomenología trascendental, es la razón de ser de estas. Por ello es fundamental manifestar que el proceso que se siga en este caso debe ser flexible. Al respecto, se empleó la entrevista como técnica de recolección de la información y como instrumento se asumió una entrevista en profundidad, la cual según Leal (2013) se despliega precisamente con el fin de que los informantes consultados suministren datos que se vinculen con el tópico abordado en la investigación.

\section{RESULTADOS}

\section{Etapa previa. Clarificación de los presupuestos}

En esta etapa de la investigación se pretendió explicitar los presupuestos esenciales relacionados con el tema de investigación. Según Leal (2008) en esta primera etapa se echan las bases para el logro del momento fenomenológico de la siguiente etapa (momento psicológico) ya que el acto de explicitar estos presupuestos como paso previo, permite al investigador ponerlos entre paréntesis, es decir, practicar la epojé fenomenológica (ver Tabla 1).

Debido a que Husserl rechaza que la conciencia no puede estar constituida por ideas sobre ideas, es necesario partir de algunos presupuestos producto de una revisión bibliográfica y algunos elementos conceptuales claves:

1. El desarrollo vocal depende del progreso del canto, que es condicionado por las técnicas de respiración, del apoyo del sonido y del medio.

2. El aprendizaje de sonidos es más efectivo cuando existe una constante asesoría y acompañamiento.

3. La eficacia del aprendizaje dependerá de un mayor tiempo de práctica inteligente que propicie así los procesos sensorio-motrices, vocales y auditivos.

4. Las dificultades evidenciadas en cuanto al desarrollo de la audición están vinculadas a la imposición de la teoría y de la escritura musical como prescriptivas del acto de audición.

5. Un proceso de pedagogía del lenguaje musical acertado podrá favorecer la musicalidad intrínseca y las experiencias previas de los estudiantes para encontrar canales intersubjetivamente validados para la comunicación de la experiencia musical, más que como imposiciones normativas. 
Tabla 1. Etapa previa del método fenomenológico, para abordar la experiencia de los estudiantes de educación musical

\begin{tabular}{llll}
\hline \multicolumn{1}{c}{ Objetivos } & \multicolumn{1}{c}{$\begin{array}{c}\text { Momento } \\
\text { fenomenológico }\end{array}$} & \multicolumn{1}{c}{ Tipo de reducción } & \multicolumn{1}{c}{$\begin{array}{c}\text { Acciones del } \\
\text { investigador }\end{array}$} \\
\hline $\begin{array}{l}\text { Revisar, clarificar y } \\
\text { explicitar los } \\
\text { presupuestos de los } \\
\text { cuales partió la } \\
\text { investigación. }\end{array}$ & $\begin{array}{l}\text { Se echaron las bases del } \\
\text { momento psicológico de }\end{array}$ & $\begin{array}{l}\text { Primera reducción } \\
\text { fenomenológica. Se puso entre } \\
\text { paréntesis (se redujo) }\end{array}$ & $\begin{array}{l}\text { Explicitó los } \\
\text { presupuestos y } \\
\text { procedió a } \\
\text { redactarlos. }\end{array}$ \\
$\begin{array}{l}\text { Reconocer la } \\
\text { importancia de los } \\
\text { presupuestos en el curso } \\
\text { de la investigación }\end{array}$ & & $\begin{array}{l}\text { Lo teórico previamente } \\
\text { establecido respecto a los }\end{array}$ & \\
& & $\begin{array}{l}\text { tópicos de la audipercepción y } \\
\text { la entonación. }\end{array}$ & \\
& & & \\
\end{tabular}

Fuente: Leal (2008).

\section{Etapa descriptiva. Momento fenomenológico psicológico}

La recolección de la información en la investigación fue lograda a través de entrevistas que según Taylor y Bogdan (como se citó en Leal, 2008) son encuentros concebidos cara a cara y que son dirigidos hacia la comprensión de las experiencias vividas por los informantes, en este caso, las vivencias de los estudiantes de educación musical en los cursos de lenguaje y percepción musical (ver Tabla 2).

Tabla 2. Etapa descriptiva del método fenomenológico, para abordar la experiencia de los estudiantes de educación musical

\begin{tabular}{|c|c|c|c|}
\hline Objetivos & $\begin{array}{c}\text { Momento } \\
\text { fenomenológico }\end{array}$ & Tipo de reducción & $\begin{array}{l}\text { Acciones del } \\
\text { investigador }\end{array}$ \\
\hline $\begin{array}{l}\text { Realizar la } \\
\text { observación } \\
\text { fenomenológica. }\end{array}$ & $\begin{array}{l}\text { Momento Psicológico } \\
\text { Se tienen datos } \\
\text { psicológicos de sujetos }\end{array}$ & $\begin{array}{l}\text { Segunda reducción } \\
\text { fenomenológica. Se } \\
\text { pusieron entre paréntesis } \\
\text { (se redujeron) }\end{array}$ & $\begin{array}{l}\text { Transcribió las } \\
\text { respuestas de los } 4 \\
\text { informantes }\end{array}$ \\
\hline \multirow{2}{*}{$\begin{array}{l}\text { Reflejar las } \\
\text { experiencias de los } \\
\text { estudiantes de } \\
\text { educación musical de } \\
\text { la manera más fiel } \\
\text { posible. }\end{array}$} & $\begin{array}{l}\text { empíricos; los } \\
\text { estudiantes de } \\
\text { educación musical } \\
\text { consultados. }\end{array}$ & $\begin{array}{l}\text { Referencias témporo- } \\
\text { espaciales } \\
\text { Experiencias vivenciales }\end{array}$ & $\begin{array}{l}\text { Identificó las } \\
\text { Expresiones de } \\
\text { conciencia de los } \\
\text { estudiantes antes } \\
\text { señalados. }\end{array}$ \\
\hline & & & $\begin{array}{l}\text { Construyó la } \\
\text { descripción } \\
\text { protocolar }\end{array}$ \\
\hline
\end{tabular}

Fuente: Leal (2008). 
Según Leal (2013) este tipo de entrevistas enfatizan en la importancia de las vivencias humanas, como fuentes válidas para la construcción de conocimientos científicos, que permitan comprender la vida del individuo en contexto.

Además la entrevista realizada fue enfocada, ya que según Leal (2013) este tipo de entrevistas se lleva a cabo con individuos concretos, y señalados previamente como informantes claves.

A continuación se exponen las transcripciones de algunos fragmentos claves de cada una de las entrevistas realizadas, y que muestran significados esenciales en las expresiones de conciencia de los informantes, las cuales corresponden a la etapa descriptiva y según Leal (2008) a un momento fenomenológico psicológico, al tenerse datos psicológicos de sujetos empíricos.

\section{Informante A}

En lo particular pienso que $\underline{\mathbf{m i}}$ experiencia ha sido excelente ya que venía de estudiar en un conservatorio (1) pero tenía mucho tiempo retirada, sin embargo cuando comencé a estudiar mis primeros semestres en el IPM pude afianzar muchísimas cosas, aprender nuevos contenidos y reforzar muchas otras que tenía olvidadas, gracias a la paciencia, la pedagogía y la dedicación de nuestro profesor (2), quien nos proporcionaba la información de forma didáctica y empleando un lenguaje simple, coherente y adecuado para la fácil comprensión de los contenidos (2).

Debo decir que siempre se me ha hecho muy fácil entonar y gracias a ello nunca he tenido dificultades para el solfeo, sin embargo considero que a pesar de poseer buenas cualidades auditivas $y$ vocales reforcé muchísimo mi entrenamiento auditivo gracias a las actividades grupales (3) y a las actividades realizadas en la institución ya que a veces en casa no es tan simple estudiar o en muchas oportunidades no contamos con el tiempo para hacerlo (4)

En cuanto a los procesos los profesores han reforzado no solo la entonación con la práctica del solfeo entonado, dictados melódicos, la rítmica (5), sino nuestro desarrollo y entrenamiento auditivo, esto desde que iniciamos, todos los cursos se han prestado de una u otra forma para que podamos reforzar estas cualidades $y$ ampliarlas aún más llevándolas a la practica en el aula de clases.

Considero que somos muy afortunados por contar con grandes profesores, con un sentido humanista y pedagógico extraordinario, siempre hay algunos casos especiales con respecto a la ética (6) de los profesores pero en realidad son la minoría. Respecto a los conocimientos de cada uno de ellos no tendríamos nada de qué quejarnos, manejan perfectamente los contenidos y son autónomos en sus áreas. Con respecto a las actividades me parece que son adecuadas 
para el nivel y los conocimientos de los estudiantes, sin embargo haría el curso un poco más didáctico, más interactivo, de forma que los estudiantes se vean en la necesidad de participar incluso de forma corporal (7), que los ayude a soltarse un poco más y a ser más seguros $y$ extrovertidos, ya que muchos llegan siendo muy tímidos y les cuesta invocarse en las actividades (8). Pienso que este aspecto es importante ya que como docentes debemos poseer seguridad para poder dirigir un curso.

\section{Informante B}

Los cursos de Lenguaje y Percepción Musical a nivel personal fueron de gran provecho y aprendizaje, pues a nivel personal llegué a la universidad de cero en cuanto a conocimientos musicales básicos, como figuras de notas musicales, rítmica, solfeo y conocimientos teóricos musicales, conocimientos que durante estos dos cursos pude conocer y poner en práctica, y que de igual manera han sido de gran provecho dentro de mi carrera.

En cuanto a mi audición y solfeo entonado se puede notar gran mejoría en comparación a mis primeros días dentro de la universidad, esta mejoría se ha dado gracias a las actividades que realizamos dentro del aula de clases durante esos cursos de Lenguaje y Percepción Musical (1). más sin embargo esta también se complementó por las diversas practicas realizadas en casa enviadas por los profesores (2) durante esos cursos, pues en el aula se practicó el solfeo en diversas oportunidades pero no lo suficiente, de ser así la mejoría hubiese sido mucho más grande (3). No tengo alguna queja sobre los profesores y su actuación pedagógica en cuanto a mi aprendizaje en ese momento, pues siempre encontraban la manera de hacerme entender o comprender lo que ameritaba el momento, pues aunque venia de cero en cuanto a mi formación musical, lograron integrarme al grupo de lenguaje en el que habian personas un poco más avanzadas musicalmente en cuanto a la lectura, y la teoría musical (4).

En cuanto a los aportes que pudiese dar en opinión personal a estos cursos de Lenguaje y Percepción Musical, quizás sería la práctica de Solfeo Entonado; ósea la asignación de más horas a estos cursos y la creación de espacios didácticos dentro de los mismos solo para Solfear (5), esto debido a que el Solfeo debe ser practicado constantemente para que pueda haber mejoría en el mismo, de no ser así, más complicado será realizar la lectura de una partitura de forma correcta, pues no se posee una práctica significativa que ayude a la misma.

\section{Informante C}

Considero que los objetivos fueron logrados porque aprendi cosas nuevas gracias a las que ya sabia (1). En cuanto a la comprensión el docente se explicaba muy bien, (al menos el profesor con quien yo vi la materia), uno salía satisfecho de clase porque había entendido el contenido (2). En cuanto a la adquisición de 
habilidades hablare en general creo que todos obtuvimos algo, muchos empezaron "chuecos" y al día de hoy ya entonan mejor, por ejemplo.

Considero que mi audición y solfeo entonado están bien, no es un excelente porque no leo una partitura extraordinariamente a la primera sin embargo con practica y tiempo todo es posible (3). Mis resultados favorables han sido por una combinación de ambos tanto las actividades en clase como el estudio en casa (4). En clase el profesor da el material y nos ayuda a realizarlo de forma correcta a su vez da herramientas para que estas actividades sean logadas satisfactoriamente luego en casa se pule lo aprendido en clase.

Tomando en cuenta el profesor que me dio esta materia, para mi fue excelente, tenía mucha paciencia, explicaba muy bien, si uno no entendía volvía a explicar sin problema, y se llevaba el curso de forma amena. Considerando que el curso de Lenguaje y Percepción Musical es una materia fundamental de nuestra especialidad vimos temas que no estaban plasmados en el plan de contenido, lo cual hace que vayamos a otro nivel con buena base (5). El que fue mi docente en esta materia me enseñó que dar una clase no solo consiste en pararse frente a estudiantes $y$ explicar, Aparte de formar también se debe educar ósea un profesor de esta era debe estar pendiente de cada detalle dentro del aula, como de sus estudiantes para así ayudarlos a que mejoren y sigan adelante (6).
En cuanto a aportes de actividades, puede ser una opción que al menos 3 clases sean fuera del aula habitual, en nuestro alrededor existen muchos sonidos, con diferentes colores, timbres, etc. (7), y que podamos aprender entre todos. Un estudiante de música debería o sería bueno que aprenda a oirlos esto haría que el odio melódico y rítmico se desarrolle y este "activo". A su vez se puede usar recursos tecnológicos pues tenemos esta herramienta muy valiosa a la cual se le puede sacar provecho, se puede proyectar un video y de alli se saca alguna dinámica o actividad evaluativa (8).

\section{Informante D}

En relación a mi experiencia puedo decir que fue buena, porque cuando uno hace lo que le gusta debe demostrar interés, y es con el interés que se buscan los logros (1). En cuanto a la comprensión no voy a decir que fue fácil porque no tenía estudios previos al llegar al instituto, pero a través de mucho estudio fui comprendiendo más y más (2). Considero que he adquirido las habilidades básicas en un buen nivel, pero no puedo decir que he adquirido destrezas a nivel macro, por ejemplo en el solfeo, tampoco lo hago como algunas personas que lo hacen muy natural y excelente.

Aquí vuelvo a decir, que mi solfeo es aceptable, sin embargo no es excelente, pues es dificil leer y solfear una partitura a primera vista, en cambio estudiándola es más fácil. Los resultados que he obtenido 
han sido gracias a mucha práctica (3) y a algunos consejos que han dado algunos profes y algunos compañeros. En el aula es más fácil porque está el profesor, pero en casa es más complicado, porque uno tiene que valerse por uno mismo (4) y a veces no tiene los recursos o estrategias necesarias para avanzar más rápido. Yo me centraba en estudiar las lecciones vistas en clase para consolidarlas y así fui memorizando algunas notas de la escala (5).

Los profesores que tuve hacian un buen trabajo porque se esforzaban en lograr que los estudiantes comprendieran, explicaban una y otra vez, y se preocupaban por los avances (6), sin embargo cuando alguien no conseguía los resultados que se esperaban creo que no era solo responsabilidad del estudiante, porque considero que los profesores deben tratar de motivar a todos, porque a veces las clases eran muy monótonas y bajo la misma modalidad (7) y eso a lo mejor a algunos no les parecía atractivo. Por eso yo creo que las clases deben ser un poco más dinámicas que haya variación de actividades (8) y que se aprenda entre todos yo creo que así se lograrán mejores resultados.

\section{Etapa estructural}

Según Leal (2008) habiéndose realizado las descripciones protocolares, en la etapa estructural se procede a su estudio y análisis con el fin de encontrar en ellas significados esenciales. Para el logro de este objetivo se desarrollaron los siguientes pasos:

Paso 1: Lectura de las descripciones de conciencia a fin de tener una primera visión del tema central al cual cada una de ellas se refiere.

Paso 2: Una segunda lectura de las cuatro descripciones protocolares para identificar en cada una de ellas el tema central. Según Leal, este segunda lectura debe realizarse respondiendo a las interrogantes ¿Qué es lo que aborda el estudiante en esta descripción protocolar? y ¿De qué trata esta descripción protocolar?

En este paso corresponde el momento fenomenológico de lógica del significado ya que en él se emplea la lógica para llegar a los temas centrales. Además se realiza una reducción fenomenológica eidética ya que se pasa de la esfera meramente fáctica (descripciones protocolares) a la esfera eidética (temas centrales) (Leal, 2008).

A continuación de muestran los resultados de este segundo paso en la Tabla 3: 
Tabla 3. Descripciones protocolares

Informante A Informante B

1. Importancia del conocimiento previo

2. Necesidad de pedagogía y didáctica en la enseñanza pertinencia del uso del lenguaje

3. Entrenamiento auditivo basado en el aprendizaje colaborativo.

4. Falta de tiempo para trabajar en casa.

5. Desarrollo de audición a través de dictados melódicos.

6. Docentes humanistas y éticos.

7. Ausencia de dinamismo y actividades prácticas corporales.

8. La timidez como barrera en la comunicación didáctica.
1. Consolidación de habilidades producto de la práctica en el aula.

2. Necesidad de prácticas constantes en casa

3. Necesidad de mayor tiempo en sesiones de clase didácticas.

4. Importancia del apoyo del docente.

5. La importancia de la práctica en contraposición a la teoría.
Informante C

1. Importancia del conocimiento previo.

2. Importancia de la Buena actuación del docente y su forma de comunicarse.

3. Desarrollo de habilidades a través del tiempo y la práctica.

4. Importancia de la integración de las actividades en aula y actividades en casa.

5. Bases sólidas para la consolidación de aprendizajes más complejos.

6. La importancia de profesores constructivistas.

7. Necesidad de Variación de escenarios de aprendizaje.

8. Importancia de las TICS en la enseñanza y el aprendizaje de la música.
Informante D

1. Interés y motivación para el logro.

2. Importancia del conocimiento previo.

3. Importancia de la práctica constante en el logro de objetivos.

4. La importancia del apoyo en clase.

5. Necesidad de estrategias para el trabajo en casa.

6. Importancia del docente como guía.

7. Monotonía y exceso de clases magistrales.

8. Necesidad de actividades prácticas dinámicas.
Paso 3: En este paso se procedió a agrupar los temas centrales por similitud. En este tercer paso, según Leal (2008) corresponde un momento fenomenológico de lógica del significado. Así como también un momento fenomenológico constitutivo trascendental, por el establecimiento de la correspondencia entre los temas centrales, para dar paso a los temas esenciales.

Además se realiza, siguiendo a Leal (2008) una reducción fenomenológica eidética ya que se pasa de la esfera meramente fáctica (temas centrales) a la esfera eidética (temas esenciales). 
A continuación de muestran los resultados de este segundo paso:

\section{Temas esenciales}

1. Importancia del conocimiento previo en el desarrollo de la audiopercepción.

2. El docente como pieza fundamental y guía del proceso de aprendizaje.

3. Necesidad de actividades didácticas en contraposición a la enseñanza conductista.

4. La práctica constante como base para el logro de los objetivos.

5. Estrategias de aprendizaje para el desarrollo de la audiopercepción en casa.

6. Importancia de actividades prácticas corporales y vivenciales.

7. Importancia del aprendizaje colaborativo.

8. Importancia de la práctica en contraposición a la teoría.

9. Docentes humanistas y éticos.

Paso 4: En este cuarto paso se procedió a la búsqueda de esencias de significado de los 9 temas esenciales surgidos. Para ello se agruparon nuevamente por similitud de contenido. A este resultado se le denomina según Leal (2008) categorías fenomenológicas esenciales/individuales/sintetizadas.

A este paso le corresponden dos momentos fenomenológicos: a) lógica del significado, por el empleo de la lógica para pasar de los temas esenciales a las categorías fenomenológicas esenciales/ individuales/sintetizadas, y b) constitutivo trascendental por la búsqueda de correspondencia entre los temas esenciales.

La reducción fenomenológica fue la eidética ya que se pasó de la esfera meramente fáctica, representada por los temas esenciales, a la espera eidética, representada por las categorías fenomenológicas esenciales/individuales /sintetizadas.

A continuación se presentan los resultados de este paso:

\section{Categorías fenomenológicas esenciales/ individuales/sintetizadas}

1. Conocimiento previo: Categoría que agrupa cada una de las respuestas relacionadas con la importancia de la experiencia y los conocimientos previos en la formación musical.

2. El docente: Categoría que contiene todas y cada una de las respuestas que muestran al docente como el principal responsable en el proceso de aprendizaje y adquisición de habilidades.

3. La práctica: Acá se agrupan las respuestas relacionadas con la necesidad y la importancia de actividades prácticas y el estudio para el correcto desarrollo de la 
audiopercepción y la memoria melódica.

\section{Aprendizaje colaborativo: Categoría} que agrupa las respuestas relacionadas con la importancia del apoyo entre los estudiantes y el docente para el logro de los objetivos.

\section{Didáctica y estrategias: Categoría que} agrupa todas las respuestas relacionadas con la importancia de la utilización de actividades didácticas que logren la vivencialidad de los procesos y además desarrolle los procesos metacognitivos y las estrategias de aprendizaje.

Paso 5: En este quinto paso se procedió a la reagrupación por similitud de las categorías fenomenológicas esenciales/individuales/sintetizadas, para así dar paso a las categorías fenomenológicas esenciales/universales (ver Tabla 4).

Tabla 4. Temas esenciales y categorías fenomenológicas

\begin{tabular}{ccc}
\hline Temas esenciales & $\begin{array}{c}\text { Categorías fenomenológicas } \\
\text { esenciales/individuales/ } \\
\text { sintetizadas }\end{array}$ & $\begin{array}{c}\text { Categorías } \\
\text { fenomenológicas } \\
\text { esenciales/universales }\end{array}$ \\
\hline
\end{tabular}

Importancia del conocimiento previo.

Necesidad de pedagogía y didáctica en la enseñanza pertinencia del uso del lenguaje.

Entrenamiento auditivo basado en el aprendizaje colaborativo.

Ausencia de dinamismo y actividades prácticas corporales.

La timidez como barrera en la comunicación didáctica.

Importancia de la integración de las actividades en aula y actividades en casa. Necesidad de Variación de escenarios de aprendizaje.

Importancia de las TICS en la enseñanza y el aprendizaje de la música.

Necesidad de estrategias para el trabajo en casa.

Necesidad de actividades prácticas dinámicas.

Monotonía y exceso de clases magistrales.

Falta de tiempo para trabajar en casa. Desarrollo de audición a través de dictados melódicos.
Aprendizaje colaborativo. Pedagogía de la música.

Didáctica y estrategias.

Conocimiento previo.

La práctica.
La práctica desde la experiencia 


\begin{tabular}{ccc}
\hline Temas esenciales & $\begin{array}{c}\text { Categorías fenomenológicas } \\
\text { esenciales/individuales/ } \\
\text { sintetizadas }\end{array}$ & $\begin{array}{c}\text { Categorías } \\
\text { fenomenológicas } \\
\text { esenciales/universales }\end{array}$ \\
\hline
\end{tabular}

Consolidación de habilidades producto de la práctica en el aula.

Necesidad de mayor tiempo en sesiones de clase.

Necesidad de prácticas constantes en casa.

La importancia de la práctica en contraposición a la teoría.

Desarrollo de habilidades a través del tiempo y la práctica.

Bases sólidas para la consolidación de aprendizajes más complejos.

Docente mediador

Importancia del conocimiento previo.

Docentes humanistas y éticos.

Importancia del apoyo del docente.

Importancia de la Buena actuación del

docente y su forma de comunicarse.

Importancia del apoyo del docente.

La importancia de profesores

constructivistas.

Interés y motivación para el logro.

La importancia del apoyo en clase.

Importancia del docente como guía.

En este paso, según Leal (2008) los momentos fenomenológicos que corresponden son (a) lógica del significado, representado por el empleo de la lógica para llegar a las categorías fenomenológicas esenciales universales; (b) constitutivo trascendental, por la búsqueda de correspondencia entre las categorías fenomenológicas esenciales/ individuales/sintetizadas y las categorías fenomenológicas esenciales/universales; y (c) metafísico de la conciencia, por la comprensión como un todo.

El tipo de reducción fenomenológica corresponde a la reducción trascendental, ya que se pasa de la universalidad fáctica a la universalidad trascendental (Leal, 2008). 
A continuación se presentan los resultados de este paso:

\section{Categorias fenomenológicas esenciales/ universales}

1. Pedagogía de la música: Métodos innovadores en los procesos de enseñanza de la música que se centran en la generación de espacios didácticos vivenciales que potencian el aprendizaje estratégico (ver Figura 1).

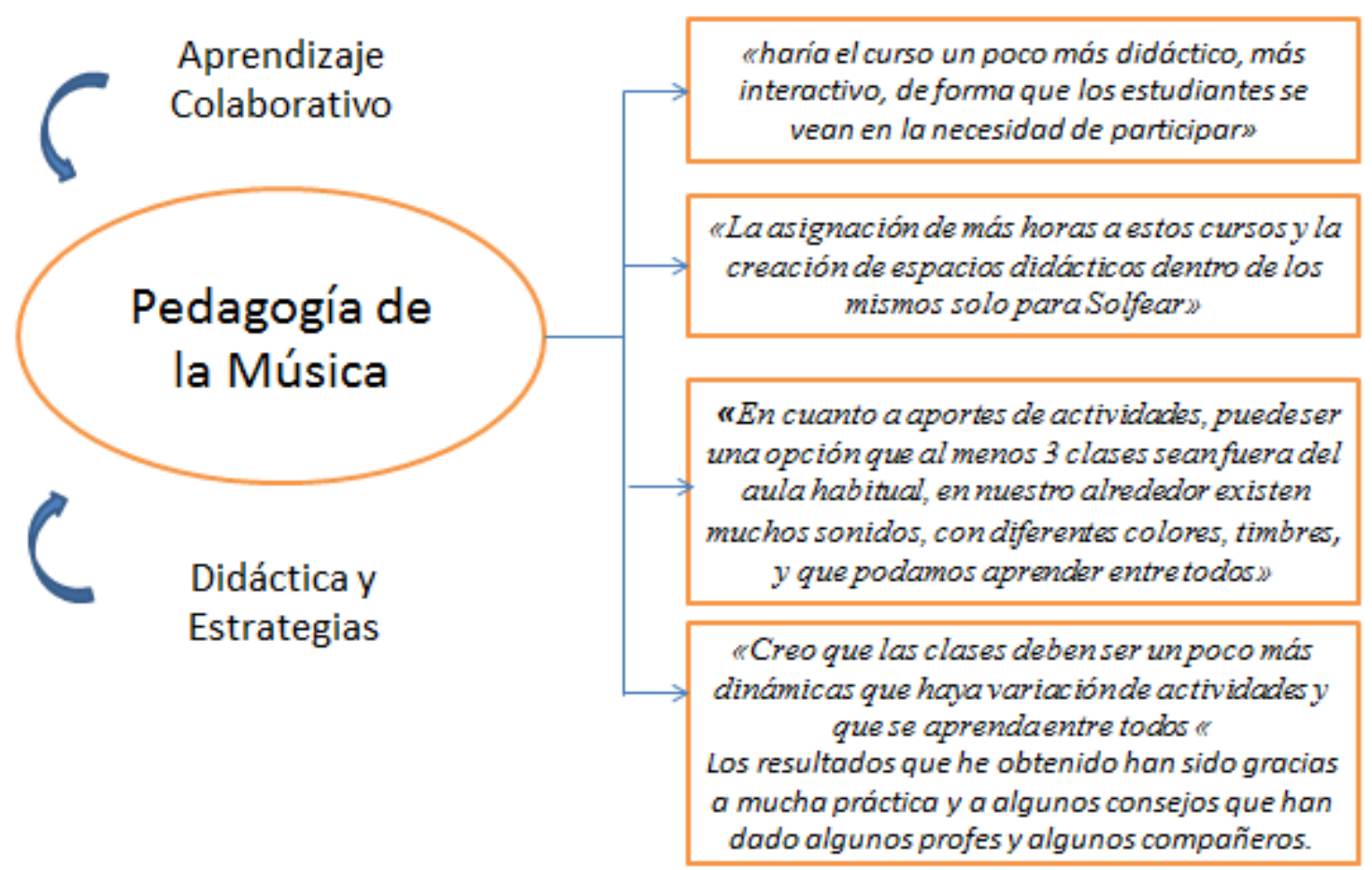

Figura 1. Pedagogía de la música.

2. La práctica desde la experiencia: La práctica musical vista desde un espacio constructivista colaborativo en donde las experiencias sirvan como andamiaje y así sustituir las prácticas no productivas (ver Figura 2). 


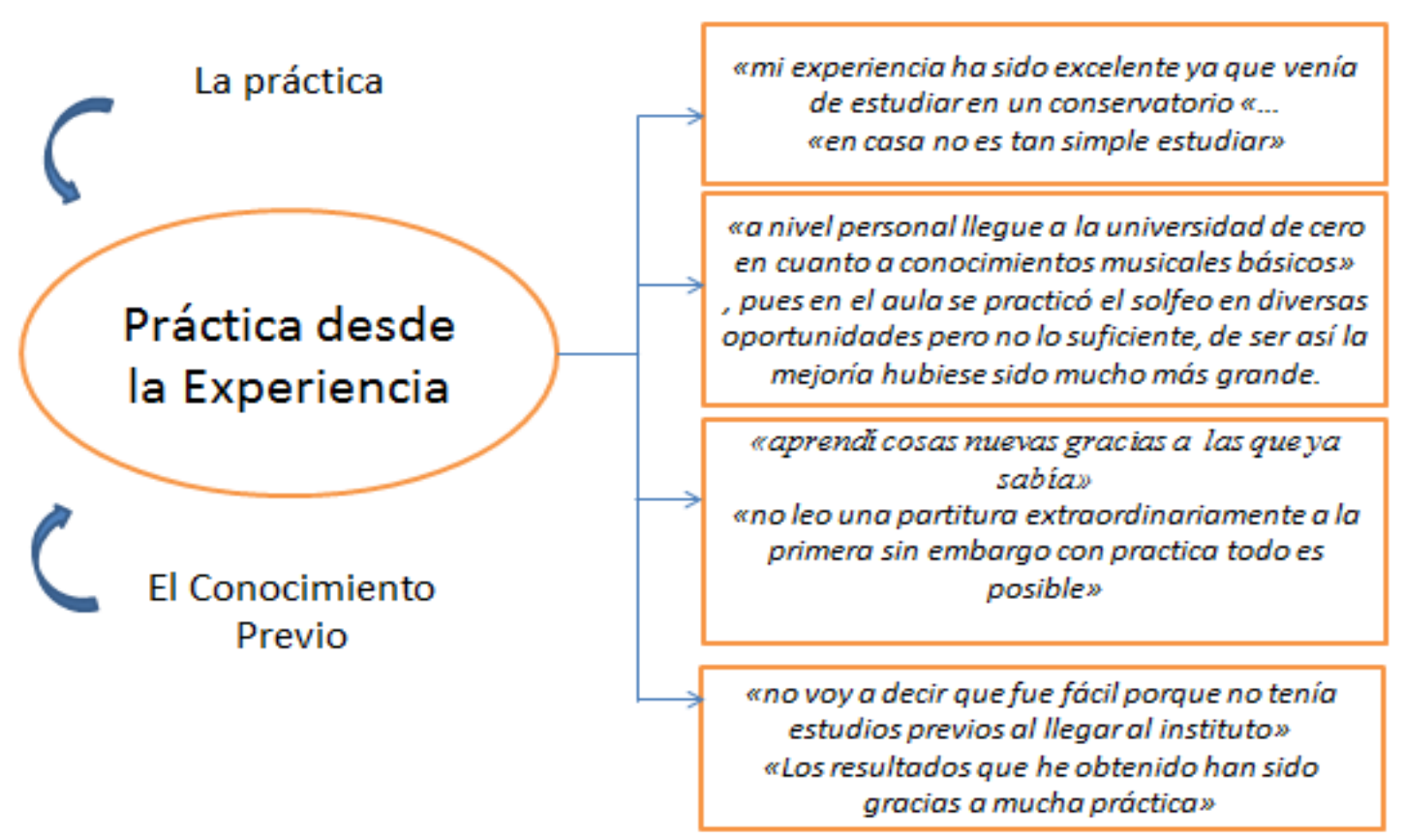

Figura 2. Práctica desde la experiencia.

3. Docente mediador: Entendido como un mediador de los aprendizajes capaz de involucrarse con los estudiantes y guiar un proceso afectivo y asertivo (ver Figura 3).

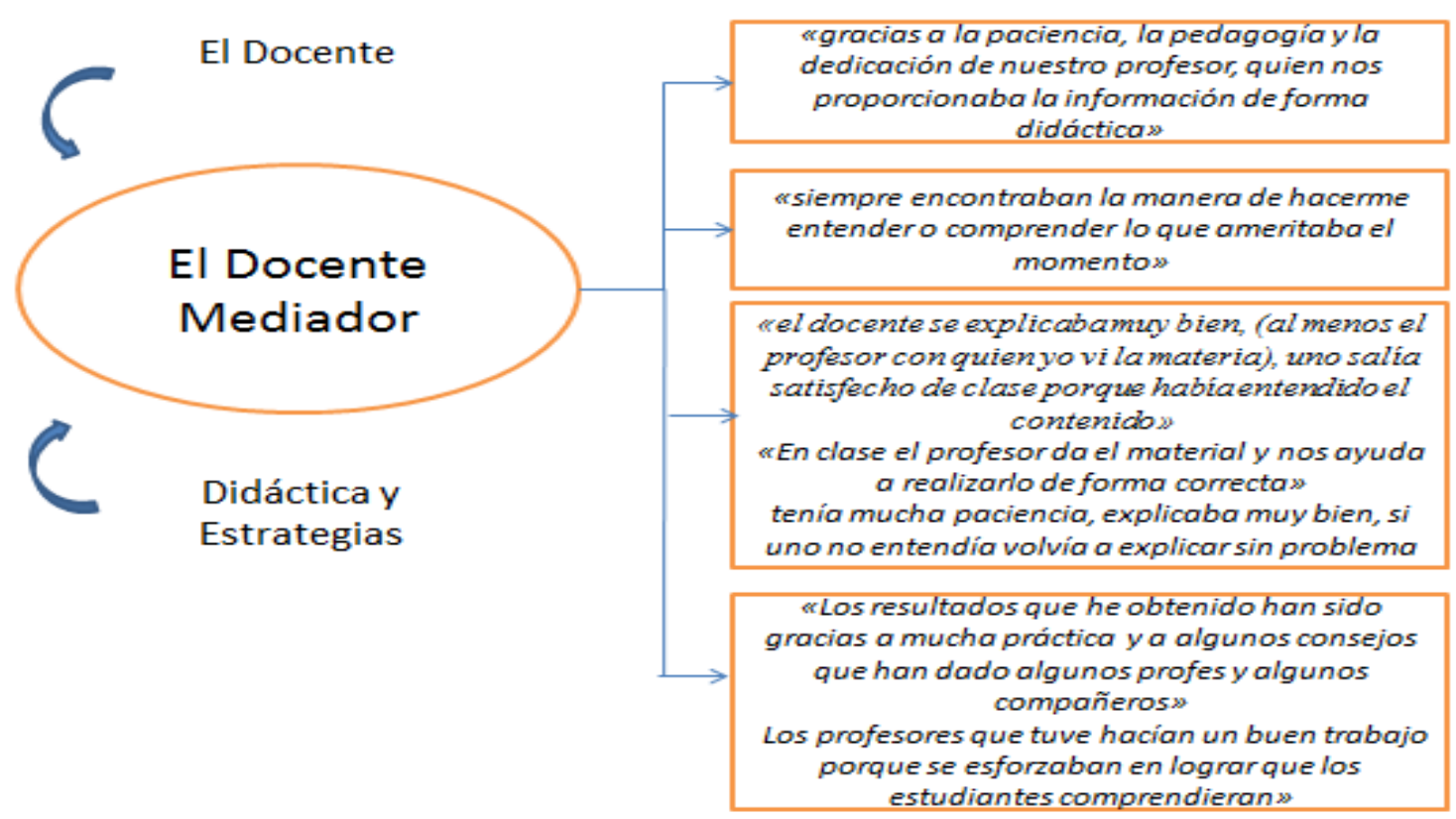

Figura 3. E1 docente mediador. 


\section{Revisión bibliográfica}

El punto de partida en una investigación basada en el método fenomenológico trascendental debe iniciar según Leal (2001) con una exhaustiva revisión bibliográfica que pretende abordar los elementos esenciales propios de la investigación, con los cuales, se generará al final de la investigación el momento fenomenológico histórico crítico.

A continuación se muestran algunos referentes teóricos relacionados con el proceso del desarrollo de la memoria auditiva y la entonación.

Colombati (2007) realizó una investigación acerca de la entonación y su de proceso de formación en la música $\mathrm{y}$ en la vida. En el proceso de investigación, las fuentes fueron revelando acontecimientos sociales y políticos importantes a nivel cultural y musical de la Unión Soviética en la primera mitad del siglo XX. Y a través de ellos se pudo establecer una perspectiva estética original y un detallado análisis de la teoría estético-musical de la entonación. El autor concluyó que las formas vocales dependen del progreso del canto que es condicionado por la manera de la respiración, del apoyo del sonido y del medio, además de recalcar la imperiosa necesidad en la expresión de las emociones que libera la cultura en la personalidad.
Piñeros y Salazar (2008) a través de una investigación sobre la pedagogía vocal lograron generar propuestas de estrategias para resolver dificultades de entonación. Para el desarrollo de esta investigación se propuso determinar el impacto de la aplicación de estrategias de pedagogía vocal a dos grupos a los cuales en varias sesiones se les aplicarían las estrategias propuestas. Los resultados obtenidos arrojaron que el estudio y el aprendizaje de sonidos son más efectivos cuando existe una constante asesoría y acompañamiento por parte del docente hacia los estudiantes; además se evidencia que la eficacia del aprendizaje dependerá también de un mayor tiempo de práctica inteligente. Se propicia así automatizar los procesos sensoriomotrices, vocales y auditivos

Shifres (2009) desarrolló una investigación que buscó analizar tres supuestos implícitos en la práctica tradicional de formación del oído musical en las instituciones especializadas y confrontarlas con las experiencias posmodernas de escucha, identificando los puntos que parecen ser incompatibles entre ambas. Gracias a la investigación se logró determinar que las dificultadas evidenciadas en cuanto al desarrollo de la audición podrían estar vinculadas a la imposición de la teoría y de la escritura musical como prescriptivas del acto de audición. A su vez el estudio sugiere 
una pedagogía del lenguaje musical que favorezca la musicalidad intrínseca y las experiencias previas de los estudiantes para encontrar canales intersubjetivamente validados para la comunicación de la experiencia musical, más que como imposiciones normativas.

Etapa de discusión y análisis. Momento fenomenológico histórico crítico

A través de la reducción fenomenológica intersubjetiva trascendental se presenta una comparación y análisis de los significados fenomenológicamente mostrados en los resultados con la revisión bibliográfica previamente realizada.

En los presupuestos mostrados al inicio de la investigación se evidencia que Piñeros y Salazar (2008) demuestran que el aprendizaje de sonidos es más efectivo cuando existe una constante asesoría y acompañamiento por parte del docente hacia los estudiantes. Este presupuesto se logró determinar a su vez en el desarrollo de la investigación, debido a que se determinó que una pieza fundamental en el proceso de aprendizaje musical, y el desarrollo de la audiopercepción y la entonación debe ser guiado por un constante tutor que demuestre a los estudiantes la forma correcta de apropiarse de los contenidos y el desarrollo de habilidades.
Vigotsky (1984) afirma que el camino a través de otra persona es la vía central de desarrollo de la inteligencia práctica. Estas afirmaciones permiten entender que para que exista un proceso de educativo efectivo es importante crear ambientes de aprendizaje donde la interacción entre docente-estudiante sea la base.

Otro presupuesto mostrado al inicio fue el determinado por Piñeros y Salazar (2008) que expresa que la eficacia del aprendizaje dependerá de un mayor tiempo de práctica inteligente que propicie así los procesos sensorio-motrices, vocales y auditivos, presupuesto que se evidenció en la investigación, ya que se logró determinar a la práctica conjuntamente con los conocimientos previos como la base para la adquisición de aprendizajes más complejos, es decir, un proceso de práctica desde la experiencia en donde el aprendizaje estratégico y la metacognición juegan un papel primordial.

Es decir, los estudiantes deben centrar sus formas de estudio en procesos reflexivos que busquen la consolidación de experiencias significativas. Muestra de esto lo define Monereo (2007) al aclarar que el aprendizaje estratégico delimita la importancia funcional de las estrategias de aprendizaje en el ámbito educativo ya que a través de ellas se logra que el estudiante tome en serio la temática y deje de considerarla como algo superficial. 
Es evidente que durante muchos años en la enseñanza de la música se ha estado desvinculado de esta perspectiva, pues el aprendizaje del solfeo y el desarrollo auditivo se ha trabajado mediante una constate de escuchar y repetir, dejando de lado las potencialidades cognitivas del estudiante, y limitándolo a la sola instrucción de los docentes. Por tales motivos es necesario que se implanten nuevos modelos de enseñanza que potencien un estudiante crítico y reflexivo, y que además entienda de qué manera se apropia más fácilmente de los contenidos alejándose de métodos de estudio que buscan el aprendizaje mecánico.

Según Lago y González (2012) al no trabajar mirando un libro, sino desarrollando la escucha consciente y la memoria a través de propuestas eminentemente activas y lúdicas, poco a poco van ampliando su capacidad de atención y concentración. Es decir, que el estudio del solfeo y el desarrollo de la audición no puede limitarse a estudiar uno o más métodos al pie de la letra, sino más bien de recurrir a actividades novedosas que despierten el interés y que su vez formen parte de un día a día sin limitarse a las solas sesiones de clase.

Otro presupuesto declarado al inicio de la investigación fue el que expresa que un proceso de pedagogía del lenguaje musical acertado podrá favorecer la musicalidad intrínseca y las experiencias previas de los estudiantes para encontrar canales intersubjetivamente validados para la comunicación de la experiencia musical, más que como imposiciones normativas (Shifres, 2009).

Esta afirmación se logró evidenciar en la investigación, pues se develó la importancia de un proceso de pedagogía musical en contraposición a la enseñanza tradicional, que busquen además la creación de espacios pedagógicos lúdicos que fomenten actividades prácticas que potencien el pensamiento reflexivo.

Estas concepciones de la educación musical desde esta perspectiva no son consideradas como una nueva tendencia pues ya desde inicios del siglo $\mathrm{XX}$ grandes compositores musicales como Dalcroze, Orff, Shafer y Kodaly se centraron en romper los esquemas tradicionales de la enseñanza de la música para conducir a los estudiantes a una formación que se inclina hacia el sentir, y no al teorizar. Estos músicos quisieron crear ambientes de estudio en donde el cuerpo jugara un papel primordial que complementara las otras actividades instrumentales y vocales.

Una gran muestra de los procesos musicales a través del cuerpo la propone Jaques Dalcroze, músico suizo nacido en Viena $(1865$ - 1950). Para algunos autores, este músico se centra en que cualquier fenómeno musical, bien sea rítmico, melódico o armónico es objeto de representación corporal, método que sin duda alguna se centra en que el niño y la niña tomen conciencia de sus movimientos 
para luego interiorizarlos, creando así una sensibilidad musical a través de la participación motriz. Las actividades basadas en este método se planifican en espacios abiertos donde el desplazamiento y la expresión corporal libre al ritmo de la música son el eje principal, también se complementan con la ejecución de patrones rítmicos sencillos y birrítmias con el cuerpo, transformándose éste en un instrumento de percusión más en la clase de música.

Estos procesos no deben ser vistos como abstractos o inentendibles, pues no debe concebirse como un modelo centrado en la enseñanza de un instrumento, sino por el contrario, en un proceso destinado a crear bases sólidas musicales mientras se potencian en gran medida aspectos físicos, cognitivos, morfológicos y motores.

\section{CONCLUSIONES}

El aprendizaje y la enseñanza de la música en el contexto educativo es un proceso complejo, debido a la abstracción que engloba el desarrollo de diversas actividades y contenidos; por tales motivos, el impartir conocimientos musicales efectivos y que a su vez se traduzcan en una correcta apreciación y entendimiento de los mismos por parte de los estudiantes, va a depender del especialista a cargo del curso de educación musical, pues para generar aprendizajes significativos el docente de música debe estar correctamente formado musical y pedagógicamente.

En relación a ello, todas las observaciones y hallazgos obtenidos lograron brindar un panorama más claro en cuanto a las necesidades en la formación del profesor de música, ya que impera la necesidad de un modelo educativo centrado en los procesos cognitivos y en el desarrollo auditivo como primer escenario. Para el logro de estos objetivos es necesario además, que el docente de música sea capaz de crear escenarios no convencionales en donde el aprendizaje colaborativo tenga un papel protagónico, además de fortalecer la formación de estudiantes críticos y reflexivos ya que de esta manera estos interpretarán la forma correcta de aprender, lo que les brindará un mayor grado de autonomía, claro está, ésta autonomía y potenciación de un pensamiento reflexivo dependerá de un cúmulo de actividades destinadas a desarrollar una verdadera práctica reflexiva que brinde al estudiante o músico en formación la posibilidad de identificar sus errores, sus potencialidades y su grado de musicalidad.

Este tipo de enseñanza a nivel musical tendrá como meta lograr un equilibrio en la balanza, ya que se deben minimizar los procesos de enseñanza conductuales donde el estudiante practica incesantemente $\mathrm{y}$ presenta ante su profesor lo 
memorizado, esto con la finalidad de obtener una calificación, ya que la comunicación didáctica debe orientarse hacia una experiencia vivida en donde todos los acontecimientos deban sentirse y experimentarse desde la sensorialidad, de esta manera, se logra un proceso activo y reflexivo del mundo sonoro, el cual es la base para la adquisición de habilidades musicales.

Es aquí en donde se está a la vista de un espacio constructivista en donde las experiencias servirán como andamiaje y así sustituir las prácticas no productivas para dar pie a una consolidación de verdaderos conocimientos previos, los cuales serán las bases para la adquisición de conocimientos o habilidades más complejas. Este tipo de modelo educativo debe ser capaz de involucrar afectivamente al docente $y$ sus estudiantes, pues es la clave para un proceso efectivo y asertivo.

\section{REFERENCIAS}

Colomboti, C. (2007). Teoría de la entonación sobre el proceso de formación de la música en la vida y obra de Boris $V$. (tesis doctoral). Universidad Autónoma de Madrid, Madrid

Husserl, E. (1949). Ideas relativas a una fenomenología pura y una filosofía fenomenológica. México: Fondo de Cultura Económica

Lago, P. y González, J. (2012). E1 pensamiento del solfeo Dalcroziano. Mucho más que rítmica. Revista de la Facultad de Educación de Albacete, 27, 89-100.
Recuperado

de http://www.revista.uclm.es/index. php/ensayos

Leal, N. (2013). Sobre el uso del reflejo durante la entrevista en profundidad. Revista UCSAR. Investigaciones de las Ciencias Sociales, IV(7). Recuperado de http://www.ucsar.edu.ve/revista/ Revista_UCSAR_2013_1.pdf

Leal, N. (2008). Fenomenología de la conciencia del estudiante de la UNA. Fondo editorial Ipasme

Leal, N. (2001). Aproximación fenomenológica a las razones por las cuales la población femenina desea estudiar en la UNA. En Informe de investigaciones educativas, $X V(1$ y 2), 91-111

Monereo, C. (2007). Hacia un nuevo paradigma del aprendizaje estratégico: el papel de la mediación social, del self y de las emociones. Electronic Journal of Research in Educational Psychology, 5(3), 497-534

Piñeros, M. y Salazar, G. (2008). Taller de pedagogía vocal. Propuesta de estrategias para resolver dificultades de entonación. Calle 14 Revista de Investigación en el campo del arte, 2(2). Recuperado de https://revistas.udistrital.edu.co/in dex.php/c14/article/view/2919

Shifres, F. (2009). Notas para un debate sobre el rol de la audición estructural en el desarrollo de las competencias auditivas de los músicos profesionales. Músicos en Congreso. Universidad Nacional del Litoral, Santa Fe. Recuperado de https://www.aacademica.org/favio .shifres/48.pdf

Vygotsky, L. (1984). Aprendizaje y desarrollo intelectual en la edad escolar. Infancia y aprendizaje. 
Infancia y Aprendizaje: Journal for the Study of Education on Developmente, 27-28, 105-116.
Recuperado de https://dialnet.unirioja.es/servlet/a rticulo? codigo $=668448$ 